\title{
Hardware triggering: maximizing speed and efficiency for live cell imaging
}

Live cell imaging experiments now require higher speeds and more data throughput than ever before. Nikon Instruments has robust tools that enable hardware triggering of imaging devices in microscopy via direct signaling between hardware. This minimizes delays, synchronizes devices, and reduces the exposure of specimens to light. This Application Note explains how Nikon's NIS-Elements hardwaretriggering workflow operates, and details its benefits for common time-lapse acquisition routines.

A microscope imaging system is a combination of several hardware devices working together; these devices may include $X Y$ motorized stages, $Z$ focus drives, piezo fast $X Y$ or $Z$ drives, filter wheels, light sources (both epifluorescence and transmitted), and detectors. Software controls the movement of these devices, so in an ideal environment, a detector's exposure time would be the rate-limiting factor in an experiment.

Unfortunately this ideal speed cannot be achieved when software is used to control device movement. The asynchronous nature of many devices with different native speeds means software must mediate device movement and image capture through a series of commands and callbacks. Latencies, due to interaction with software, occur as a result. Latency may not be as noticeable for single images or short experiments, but the effects on the total acquisition time for large data experiments can be extremely negative.

The timing clock on the host computer ultimately dictates performance. Thus, other processes being managed on the same computer can easily affect high-precision timing.

Bypassing software latencies through direct hardware triggering in NIS-Elements

Most of today's high-performance detectors and peripherals have I/O (input and output) ports for direct voltage-mediated control of device mechanics. For example, most light sources have direct analog and TTL (transistor-transistor logic) control connectors, as well as the usual serial communications. However, commercial software packages generally use serial communication, which relies solely on the PC clock and software control.

Nikon's NIS-Elements software has been utilizing I/O connections for quite some time, and continues to expand capabilities. This software provides hardware-triggering functionality by using these

\section{Michael A Davis}

Nikon Instruments Inc., Melville, New York, USA. Correspondence should be addressed to M.A.D. (mdavis@nikon.net) direct I/O voltage-mediated connections to quickly drive devices.

Synchronization of device control with acquisition is paramount, so sensing of the detector's status (exposing or not exposing), as well as keeping count of frame number, is crucial. All of these signals are managed through digital acquisition interfaces (DAQs) for flexibility and convenience. The timing clock on a National Instruments data acquisition device (NIDAQ) is also much more precise than that of the host PC. NIS-Elements supports National Instruments NIDAQmx series devices (totaling more than 40 options) for this purpose. Because there is no requirement for software checks and callbacks in this scenario, latency is greatly reduced.

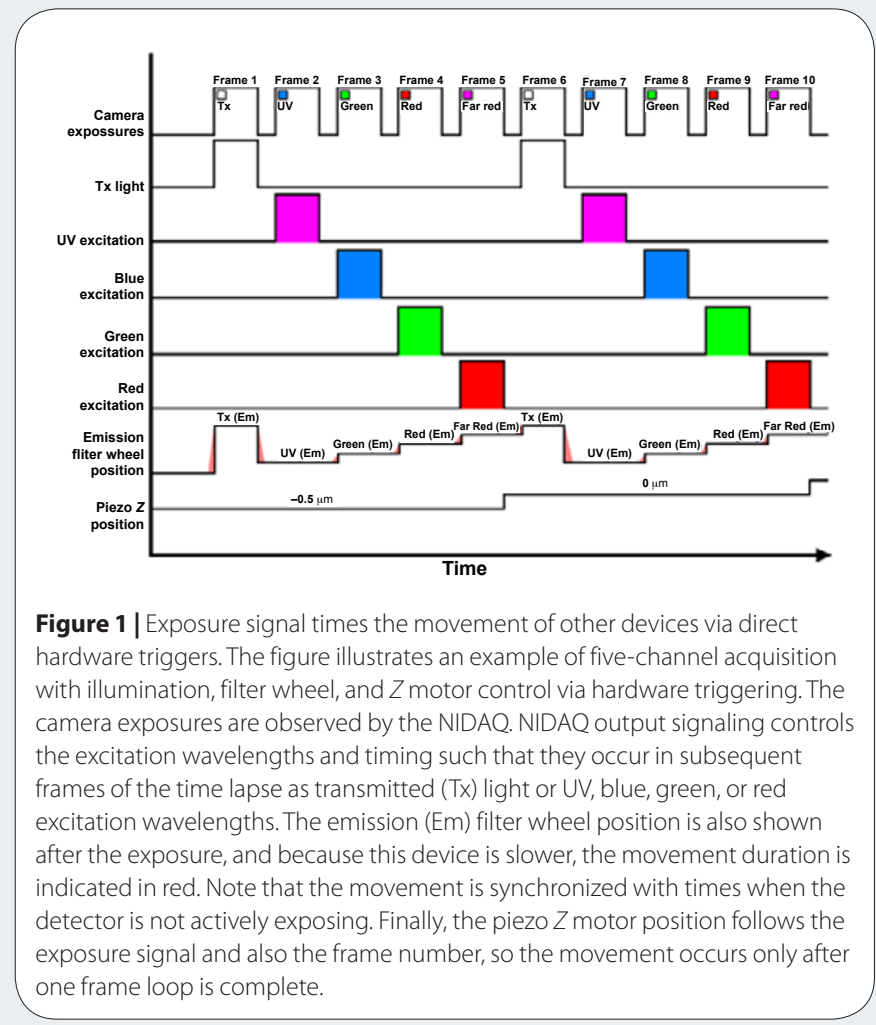




\section{How NIS-Elements performs hardware triggering}

With the detector exposure signal being observed by the NIDAQ and with peripheral hardware devices also connected, hardware triggering becomes nearly transparent. One defines multidimensional experiment parameters, and NIS-Elements sets up the sequence on the NIDAQ card. Once the detector starts, the NIDAQ moves devices between exposures and controls illumination during exposures. In this arrangement, the detector provides the master signal to the NIDAQ, and the NIDAQ orchestrates the timing and order of all other peripherals (Fig. 1).

NIDAQ allows for precise illumination control, as light sources are on only when the detector is actively exposing and immediately switch off when the camera is not exposing (including during the detector's readout time). Furthermore, NIDAQ can even customize how long the illumination should be active within an exposure, and also pulse the illumination. This control reduces the duration of illumination, keeping this time to an absolute minimum. With LED or laser sources, on/off times are in the range of tens of microseconds.

Hardware triggering also executes multiple-device control efficiently by starting all of the devices simultaneously. Parallelization improves the overall speed, as there is not a sequential execution of one device movement after another. Device timing and speed are also known, so NIS-Elements can even place device movements during the readout time of detectors running in overlapped ('live') acquisition modes, in which the detector is always running.

\section{Ti2-E: a hardware-triggered microscope stand}

Nikon Instruments expanded hardware-triggering potential with the release of the Ti2-E inverted microscope, the first commercial microscope stand in which all motorized components on the stand can take advantage of hardware triggering or latency reduction.

Key capabilities of the Ti2-E include native direct connection capabilities (even without NIDAQ) to detectors: the microscope itself knows when the exposure is complete and immediately executes the next device movements. This allows for several impressive features, including very fast autofocus routines.

Inclusion of a NIDAQ initiates the microscope hardware by direct hardware triggering as well, thereby allowing customized triggered configurations to include both microscope hardware and ancillary devices from several vendors. For example, transmitted and epifluorescence light sources, dichroic mirrors, piezo $Z$ drives, and emission filters may all be controlled together.

\section{Nikon Microscope Systems utilizes hardware triggering}

To utilize hardware triggering, NIS-Elements AR, C, and HC packages require a NIDAQ and a plugin for the triggered device control to operate.

NIS-Elements can manage a robust and growing number of hardware-triggered device types (Table 1), including light sources, motors, illumination modules, sensors, and signal generators. All of these devices can be used for basic multidimensional acquisition experiments, but are also capable of being used in stimulation/ activation experiments, high-content acquisition experiments, and custom experiment interfaces such as illumination sequencing, which gives users complete flexibility over triggered device controls, experiment order, and patterns.

\section{Table 1 | Classes of devices NIS-Elements natively controls for} hardware triggering

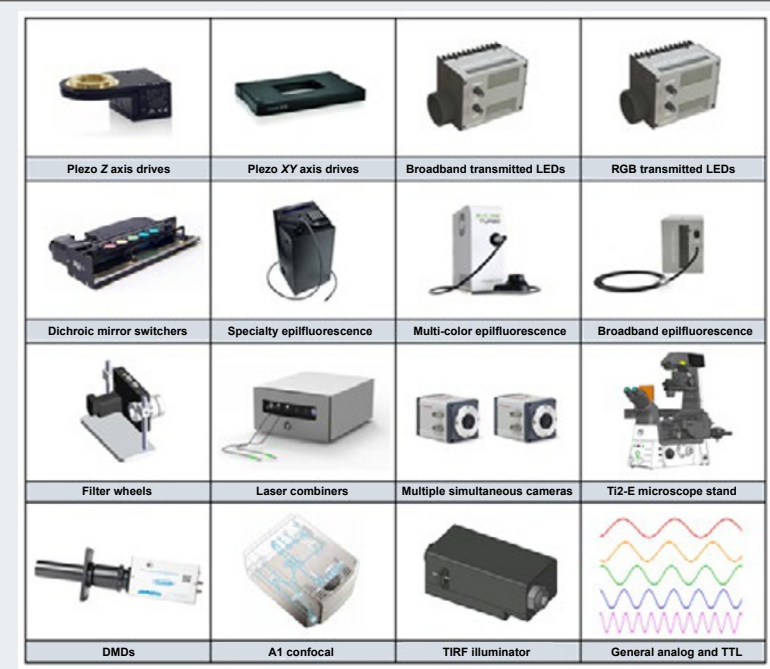

$\mathrm{DMD}$, digital micromirror device

Device configuration for hardware triggering is an easy task with peripherals like Nikon's National Instruments Breakout Box (NI-BB) package (Fig. 2), which includes a NIDAQ and a specialized breakout box with predefined connectors and cables for $X Y Z$ devices, fluorescent LEDs, transmitted light LEDs, filter wheels, laser units, photostimulation devices, and the Ti2-E triggered microscope stand. The NIS-Elements device manager simply assigns NIDAQ resources to each connected device according to the connector used. Detectors (up to three) also connect readily to the control box.

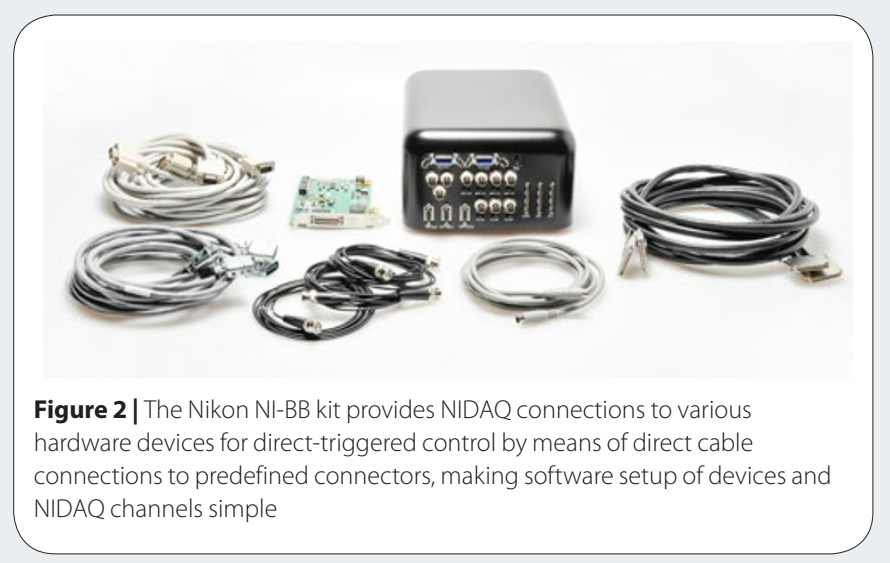

\section{Summary}

Nikon's NIS-Elements hardware triggering is a robust and effective measure to minimize the exposure of samples to light, reduce or remove latencies between exposures, and greatly decrease the overall experiment duration. Some typical experimental examples are shown in Table 2, which compares outcomes with the same hardware and 
settings. The only variable was the use of direct hardware triggering and microscope exposure end-sensing capability on the Ti2-E inverted microscope.

Table 2 | Comparison of experiment durations with typical hardware and experiment parameters

\begin{tabular}{|c|c|c|}
\hline \multirow[b]{2}{*}{ Experiment } & \multicolumn{2}{|c|}{ Experiment duration } \\
\hline & Software triggering & Hardware triggering \\
\hline $3 \lambda, 6 Z$ positions & $40 \mathrm{~s}$ & $18 \mathrm{~s}$ \\
\hline $\begin{array}{c}96 \text { stage positions, } 3 \lambda \text {, } \\
10 Z \text { positions }\end{array}$ & $12 \min 15 \mathrm{~s}$ & $6 \mathrm{~min} 21 \mathrm{~s}$ \\
\hline $\begin{array}{c}\text { Time lapse: } 10 \text { loops, } \\
3 \lambda, 15 Z \text { positions }\end{array}$ & $1 \mathrm{~min} 38 \mathrm{~s}$ & $49 \mathrm{~s}$ \\
\hline $\begin{array}{l}\text { Time lapse: } 10 \text { loops, } \\
3 \lambda, 10 Z \text { positions }{ }^{a}\end{array}$ & $1 \mathrm{~min} 13 \mathrm{~s}$ & $10.7 \mathrm{~s}$ \\
\hline \multicolumn{3}{|c|}{$\begin{array}{l}\text { Hardware triggering significantly improves the overall experiment time. In most of these examples, th } \\
\text { camera exposure time was longer than the camera readout time. } \\
\text { aThe last row presents a comparison for which the camera exposure time was the same as its readout } \\
\text { time, and the camera was able to run in overlapped ( } 100 \% \text { duty cycle) mode. }\end{array}$} \\
\hline
\end{tabular}

When investing in a microscope system, consider the peripheral devices that will be used. Devices with capabilities for I/O triggering, even if not used initially, provide a built-in upgrade pathway. NISElements software controls to enable hardware triggering are a minor expenditure, and configuration is easy. Experiment design and control with hardware triggering is both logical and streamlined, and users can save and recall triggered device configurations with the touch of a button. 